\title{
Stat5 promotes metastatic behavior of human prostate cancer cells in vitro and in vivo
}

\author{
Lei Gu ${ }^{1}$, Paraskevi Vogiatzi ${ }^{1}$, Martin Puhr ${ }^{1}$, Ayush Dagvadorj ${ }^{1}$, Jacqueline Lutz ${ }^{1}$, \\ Amy Ryder ${ }^{1}$, Sankar Addya ${ }^{1}$, Paolo Fortina ${ }^{1,2}$, Carlton Cooper $^{3}$, \\ Benjamin Leiby ${ }^{4}$, Abhijit Dasgupta ${ }^{4}$, Terry Hyslop ${ }^{4}$, Lukas Bubendorf ${ }^{5}$, \\ Kalle Alanen ${ }^{6}$, Tuomas Mirtti ${ }^{6}$ and Marja T Nevalainen ${ }^{1,7,8}$
}

\author{
${ }^{1}$ Cancer Biology, Thomas Jefferson University, Philadelphia, Pennsylvania, USA \\ ${ }^{2}$ Medicina Sperimentale e Patologia, Universita degli Studi 'La Sapienza', Rome, Italy \\ ${ }^{3}$ Biological Sciences, University of Delaware, Newark, Delaware, USA \\ ${ }^{4}$ Pharmacy and Experimental Therapeutics, Thomas Jefferson University, Philadelphia, Pennsylvania, USA \\ ${ }^{5}$ Institute for Pathology, University of Basel, Basel, Switzerland \\ ${ }^{6}$ Pathology, University of Turku, Turku, Finland \\ ${ }^{7}$ Urology and ${ }^{8}$ Medical Oncology, Thomas Jefferson University, Philadelphia, Pennsylvania, USA
}

(Correspondence should be addressed to M T Nevalainen at Cancer Biology, Urology, Medical Oncology, Thomas Jefferson University, Philadelphia, PA 19107, USA; Email: marja.nevalainen@jefferson.edu)

\begin{abstract}
There are no effective therapies for disseminated prostate cancer. Constitutive activation of Stat5 in prostate cancer is associated with cancer lesions of high histological grade. We have shown that Stat5 is activated in $61 \%$ of distant metastases of clinical prostate cancer. Active Stat5 increased metastases formation of prostate cancer cells in nude mice by 11-fold in an experimental metastases assay. Active Stat5 promoted migration and invasion of prostate cancer cells, and induced rearrangement of the microtubule network. Active Stat 5 expression was associated with decreased cell surface E-cadherin levels, while heterotypic adhesion of prostate cancer cells to endothelial cells was stimulated by active Stat5. Activation of Stat5 and Stat5-induced binding of prostate cancer cells to endothelial cells were decreased by inhibition of Src but not of Jak2. Gene expression profiling indicated that $21 \%$ of Stat5-regulated genes in prostate cancer cells were related to metastases, while $7.9 \%$ were related to proliferation and $3.9 \%$ to apoptosis. The work presented here provides the first evidence of Stat 5 involvement in the induction of metastatic behavior of human prostate cancer cells in vitro and in vivo. Stat 5 may provide a therapeutic target protein for disseminated prostate cancer.
\end{abstract}

Endocrine-Related Cancer (2010) 17 481-493

\section{Introduction}

The complex molecular mechanisms underlying the progression of prostate cancer from organ-confined tumor to metastatic disease are largely unknown. Identification of the molecular changes associated with the metastasis process of prostate cancer will provide a rational basis for the development of effective therapies for advanced prostate cancer.

Transcription factor (TF) Stat5 is critical for the growth of human prostate cancer. Stat5 belongs to the Stat gene family (Ihle 2001). The two highly homologous (98\%) isoforms of Stat5, Stat5a and Stat5b (Ihle 2001), act as both cytoplasmic signaling proteins and nuclear TFs. Stat5 becomes active by phosphorylation of a specific tyrosine residue in the carboxy-terminal domain (Ihle 2001), homo- or heterodimerizes, and translocates to the nucleus where it binds to the target gene promoters (Ihle 2001).

Stat5 is constitutively active in human prostate cancer, but not in normal prostate epithelium (Ahonen et al. 2003, Li et al. 2004). Stat5 activation is associated with high histological grade (Li et al. 2004, 2005), 
and is present in the majority of castration-resistant recurrent human prostate cancers (Tan et al. 2008). The molecular mechanisms underlying constitutive activation of Stat5 in primary and recurrent human prostate cancers are currently unclear, and may involve the autocrine Prl-Jak2 pathway (Nevalainen et al. 1997a, b, Li et al. 2004), Src kinases (Yang et al. 2009), or Rho GTPases (Benitah et al. 2003). Inhibition of Stat5 action induces rapid and massive apoptotic death of human prostate cancer cells in culture (Ahonen et al. 2003), and blocks human prostate cancer xenograft tumor growth in nude mice (Dagvadorj et al. 2008a). Stat5 positively regulates $\mathrm{Bcl}-\mathrm{X}_{\mathrm{L}}$ and cyclin-D1 protein levels in prostate cancer cells (Dagvadorj et al. 2008a), which likely underlies Stat5 induction of prostate cancer cell viability. Stat5 activation in primary prostate cancer predicted early disease recurrence (Li et al. 2005), which led us to propose that Stat5 promotes prostate cancer progression by inducing metastatic behavior of human prostate cancer cells.

Here, we have demonstrated that Stat5 is activated in $61 \%$ of distant clinical prostate cancer metastases. Stat5 activation increased migration and invasion of human prostate cancer cells, and induced a rearrangement of the tubulin network in prostate cancer cells. Active Stat5 increased metastases formation of DU145 cells in the lungs of nude mice by 11 -fold in an in vivo experimental metastases assay. Active Stat5 decreased cell surface E-cadherin expression, while heterotypic adhesion of prostate cancer cells to endothelial cells was increased by active Stat5, which was related to Src activity rather than to Jak2. Identification of Stat5-regulated genes in prostate cancer cells indicated an association of $21 \%$ of the genes with metastases processes. In summary, we have shown for the first time that active Stat5 promotes metastatic behavior of prostate cancer cells, and Stat5 may provide a therapeutic target protein for locally advanced or disseminated prostate cancer.x

\section{Materials and methods}

\section{Cell lines and clinical human prostate cancer metastases}

CWR22Rv, DU145, PC-3, and LNCaP prostate cancer cells (ATCC, Manassas, VA, USA) were grown as described previously (Dagvadorj et al. 2008a). Human umbilical cord vein endothelial cells (HUVECs; ATCC) were grown in F12K medium (ATCC), and human bone marrow endothelial cells (HBMECs) were grown in M199 medium (Sigma) as described previously (Lehr \& Pienta 1998).
Paraffin-embedded prostate cancer metastases were obtained from the Turku University Hospital and the Institute for Pathology, University of Basel $(n=128$; to lymph nodes, $n=59$; to bone, $n=15$; and to other organs, $n=54$; approved by the Thomas Jefferson University Institutional Review Board).

\section{Generation of replication-deficient adenoviruses for the gene delivery of wild-type (WT) Stat5a, WTStat5b, active Stat5 (AdStat5aS710F), dominant-negative Jak2, and WTJak2}

Plasmid cDNA-CMV-WTStat5a, pcDNA-CMVWTStat5b, and pcDNA-CMV-Stat5aS710F (Onishi et al. 1998, Moriggl et al. 2005; Supplementary Figure 1, see section on supplementary data given at the end of this article) were cloned into an adenoviral vector using BD Adeno-X Expression System 2 (BD Biosciences, San Jose, CA, USA) as described previously (Dagvadorj et al. 2008a). Viral stocks (AdWTStat5a, AdWTStat5b, AdStat5aS710F, AdLacZ, AdWTJak2, and AdDNJak2) were purified by double cesium chloride gradient centrifugation, and were titered side by side by standard plaque assay in QBI-293A cells. Adenoviruses were delivered to prostate cancer cells by incubation of the cells for 90 min with the indicated multiplicity of infection (MOI) in serum-free medium at $37^{\circ} \mathrm{C}$.

\section{Immunostaining of paraffin-embedded tissue sections}

Immunostaining was performed as described previously (Ahonen et al. 2002, 2003, Nevalainen et al. 2002, 2004). The primary antibodies were antiphospho (Y694/Y699) Stat5a/b (mAb) $(0.6 \mu \mathrm{g} / \mathrm{ml}$; Advantex BioReagents, Conroe, TX, USA) and antiStat5ab (mAb) $(2 \mu \mathrm{g} / \mathrm{ml}$; Santa Cruz Biotechnology, Santa Cruz, CA, USA). Individual prostate tumor samples were scored for active and nuclear Stat5 levels on a scale from 0 to 1 , where 0 was undetectable and 1 represented positive immunostaining. Score 1 for phosphorylated and nuclear Stat5 was used to define positive Stat5 activation status.

\section{Immunofluorescence cytochemistry of tubulin, $\alpha$-actin, and E-cadherin}

DU145, LNCaP, and PC-3 cells were infected with AdLacZ or AdStat5aS710F at MOI 10, and the cells were fixed and incubated with rhodamineconjugated phalloidin (Invitrogen, Carlsbad, CA, USA). For tubulin and E-cadherin, the cells were stained with anti-tubulin mAb (Invitrogen; 1:100) or 
anti-E-cadherin mAb (Santa Cruz Biotechnology; 1:100), followed by incubation with FITC-conjugated secondary antibodies (Invitrogen) and DAPI (blue) or propidium iodide (red; Vector Labs, Burlingame, CA, USA).

\section{Wound-filling assay}

Identical scratches were made in parallel wells $24 \mathrm{~h}$ after infection using a $1000-\mu l$ pipette tip. The cells were fixed in $0.5 \%$ crystal violet solution (Sigma) at 0 , 24 , and $48 \mathrm{~h}$.

\section{Boyden chamber cell motility and migration assay}

Single-cell suspensions were added to the upper chambers of the motility chamber system $(8.0 \mu \mathrm{m}$ pore size; BD BioSciences) $24 \mathrm{~h}$ after infection (MOI 10). For invasion assays, we employed Matrigelcoated membranes in the invasion chambers (BD BioSciences) using fetal bovine serum (FBS) (10\%) as the chemoattractant. After $24 \mathrm{~h}$, the cells that had traversed the membrane pores were fixed, stained, and counted. Each experiment was done in triplicate.

\section{Cell surface biotinylation}

Cells were grown to semiconfluence, and were biotinylated with $1 \mathrm{mg} / \mathrm{ml}$ sulfo-NHS-LC-Biotin (Pierce, Rockford, IL, USA) for $30 \mathrm{~min}$ on ice.

\section{Protein solubilization, immunoprecipitation, and immunoblotting}

Prostate cancer cell pellets were solubilized in a lysis buffer as described previously (Ahonen et al. 2002, 2003, Li et al. 2004, Dagvadorj et al. 2007, 2008a,b, Tan et al. 2008). Stat5a and Stat5b were immunoprecipitated with anti-Stat5a or anti-Stat5b pAbs (Advantex Bioreagents). E-cadherin was immunoprecipitated using anti-E-cadherin mAb (Santa Cruz Biotechnologies). For western blotting, the primary antibodies were used at the following concentrations: anti-Stat5 mAb, 1:250 (Transduction Laboratories, Lexington, KY, USA); anti-phosphotyrosine-Stat5a/b (Y694/Y699) mAb, $1 \mu \mathrm{g} / \mathrm{ml}$ (Advantex BioReagents); anti-E-cadherin mAb, 1:1000 (Santa Cruz Biotechnologies); and anti-actin, 1:4000 (Sigma). For the experiments investigating Rho GTPase and Src kinase in Stat5 activation and heterotypic adhesion, DU145 and LNCaP cells were treated with RhoA (Y27632) and Rac1 (553502) inhibitors (EMD Chemicals, Gibbstown, NJ, USA) or the Src inhibitor PP2 (EMD Chemicals) for 72 or $48 \mathrm{~h}$ at the indicated concentrations using PP3 as the control compound (EMD Chemicals).

\section{Tail vein injections of human prostate cancer cells}

Castrated male athymic mice (Taconic, Germantown, NY, USA) were implanted with dihydrotestosterone (DHT) pellets ( 1 pellet/mouse, 90 d release; Innovative Research of America, Sarasota, FL, USA) to normalize the circulating levels of DHT. DU145 cells were infected with AdLacZ or AdStat5aS710F (MOI 10). After $24 \mathrm{~h}, 1 \times 10^{6}$ cells were suspended in $0.2 \mathrm{ml}$ of PBS, and were injected into the lateral tail vein using a 27-gauge needle. After 8 weeks, the lungs were perfused with $1.5 \mathrm{ml}$ of $15 \%$ India Ink dye in $3.7 \%$ formalin, and were bleached in Fekete's destaining solution (70\% ethanol, $3.7 \%$ formaldehyde, and $0.7 \mathrm{M}$ glacial acetic acid).

\section{Adhesion assay of human prostate cancer cells to endothelial cells}

DU145 and PC-3 cells were infected with AdLacZ or AdStat5aS710F at MOI 10. After $72 \mathrm{~h}$ of adenoviral infection, the cells were stained with CytoTracker fluorescent dye (Cell BioLabs, San Diego, CA, USA). The stained prostate cancer cells were added to HUVEC or HBMEC (Lehr \& Pienta 1998) monolayers for $60 \mathrm{~min}$ at $37^{\circ} \mathrm{C}$. Prostate cancer cell adhesion to the endothelial cells was measured in a fluorescence plate reader (POLARstar OPTIMA, BMG LabTech, Cary, NC, USA). Every experimental group was repeated in sextuplicates at least three times.

\section{SiRNA transfection}

DU145 cells were transfected with scrambled siRNA or siRNAs targeted to human Stat5a and Stat5b (Dharmacon, Lafayette, CO, USA; 100 pmol of siRNA) using Lipofectamine 2000 (Invitrogen).

\section{RNA preparation and expression profiling}

Cells were harvested $48 \mathrm{~h}$ after the transfection, and total RNA was prepared using the Qiagen RNeasy Mini kit (Qiagen). A DNase I digestion step was included to eliminate DNA contamination. Both groups (control siRNA and Stat5a/b siRNA) were prepared in triplicate, and RNA quality was assessed on an Agilent 2100 Bioanalyzer (Agilent Technologies, Palo Alto, CA, USA). RNA $(2 \mu \mathrm{g})$ from each sample was used for Affymetrix one-cycle target labeling (Affymetrix, Santa Clara, CA, USA). Each Affymetrix GeneChip for Human Genome 133 Plus 2.0 was hybridized for $16 \mathrm{~h}$ with biotin-labeled fragmented cRNA $(10 \mu \mathrm{g})$ according to the Affymetrix protocol. The hybridization signals were amplified using 
antibody amplification with goat IgG (Sigma) and anti-streptavidin biotinylated antibody. Chips were scanned on an Affymetrix GeneChip Scanner 3000 using the GeneChip Operating Software version 3.0.

\section{Statistical analysis}

Chips ( $n=3$ control siRNA and $n=3$ Stat5a/b siRNA) were preprocessed using Robust Multi-array Average (Irizarry et al. 2003). The Affymetrix GeneChip for Human Genome 133 Plus 2.0 has 54675 transcripts, and nonspecific filtering (Falcon \& Gentleman 2007) was done prior to analysis, resulting in a single transcript for each of 15992 annotated genes. Probe sets which did not have an associated Entrez Gene ID and Gene Ontology (GO) ID were filtered out. The probe set which exhibited maximal variability was chosen to represent each gene (defined by a unique Entrez Gene ID). Differential expression of each gene between control siRNA and Stat5 siRNA groups was assessed using a linear model with an empirical Bayes correction for variance, as implemented in the Bioconductor (Gentleman et al. 2004) package limma (Smyth 2004). This allows a robust analysis of differential expression between control siRNA and Stat5 siRNA groups even in case of a small sample size. The $P$ values were corrected for multiple testing using the false discovery rate (FDR; Benjamini \& Hochberg 1995). This analysis establishes whether the fold change observed is large enough compared to the variability in the gene expression across the samples to meet the statistical significance criterion. We considered the genes with a FDR-adjusted $P$ value of $\leq 0.01$ to be statistically significant, which resulted in 777 genes being differentially expressed between the two groups (Supplementary Table 1, see section on supplementary data given at the end of this article, GEO accession number GSE 17483).

In Boyden chamber migration and invasion assays, endothelial cell binding assays, and in vivo metastases assay, Wilcoxon rank sum tests with exact $P$ values were used for each of the comparisons of WTStat5a, WTStat5b, and Stat5aS710F to LacZ.

\section{Results}

\section{Stat 5 is constitutively active in clinical prostate cancer metastases}

We assessed the frequency of Stat5 activation in clinical prostate cancer metastases to lymph nodes, bone, and other distant organs $(n=128)$ by immunohistochemistry (Fig. 1A, i) as demonstrated previously (Ahonen et al. 2002, Nevalainen et al. 2002, 2004,
Li et al. 2004, 2005, Tan et al. 2008). Prostate cancer metastases sections were also immunostained for prostate-specific antigen (PSA) to verify the location of prostate cancer cells within the metastasescontaining tissues, and the immunostaining for active Stat5 correlated with the PSA immunostaining in the majority of the cases. Given that $20 \%$ of bone metastases are produced by prostate cancer cells not expressing PSA (Cheville et al. 2002), the cells positive for PSA in lymph node and bone metastases should be of prostate cancer origin. Significant positive reaction for active Stat5 was detected in 78/128 (61\%) of human prostate cancer metastases (Table 1). Stat5 was activated in $48 / 59(81 \%)$ of lymph node metastases and 5/15 (33\%) of bone metastases, and in $29 / 54(46 \%)$ of prostate cancer metastases to distant organs other than bone (Table 1). In conclusion, Stat5 is active at high frequency in clinical human prostate cancer metastases to both lymph nodes and other organs.

\section{Stat5 induces migration and invasion of human prostate cancer cells}

To determine whether Stat 5 affects migration of human prostate cancer cells, DU145 and PC-3 cells were selected as the experimental models because both cell lines are androgen independent with metastatic potential in in vivo studies (Nemeth et al. 1999, Timar et al. 2000). Figure 1A, ii demonstrates Stat5a and Stat5b protein levels and their activation in various human prostate cancer cell lines. Introduction of active Stat5 (Stat5aS710F) by adenoviral gene delivery (Ad-activeStat5) at MOI 10 into PC-3 cells, which do not express Stat5, brings the active Stat5 expression in PC-3 cells to levels equivalent to those of the endogenous Stat5 expression in DU145 cells (Fig. 1A, iii).

Ad-activeStat5 (Onishi et al. 1998, Moriggl et al. 2005; Supplementary Figure 1) at MOI 10 in DU145 cells increased cell migration by $\sim 60 \%$ versus AdLacZ (MOI 10) as determined by wound-filling assay $48 \mathrm{~h}$ after adenoviral exposure (Fig. 1B, i and ii). In Boyden chamber assays, DU145 cell motility increased by $\sim 40-50 \%$ by Ad-activeStat5a after $48 \mathrm{~h}$ versus AdLacZ-infected cells $(P=0.042$; Fig. $1 \mathrm{~B}$, iii). Ad-activeStat5 increased the invasion of DU145 cells in Boyden chamber invasion assays by $\sim 100 \%$ versus $\beta$-galactosidase-expressing cells $(P=0.0029$; Fig. 1B, iv). Adenoviral expression of WT Stat5a (AdWTStat5a) or AdWTStat5b did not affect the migration of DU145 cells in wound-filling or Boyden chamber assays (WTStat5a, $P=0.91$ and WTStat5b, 


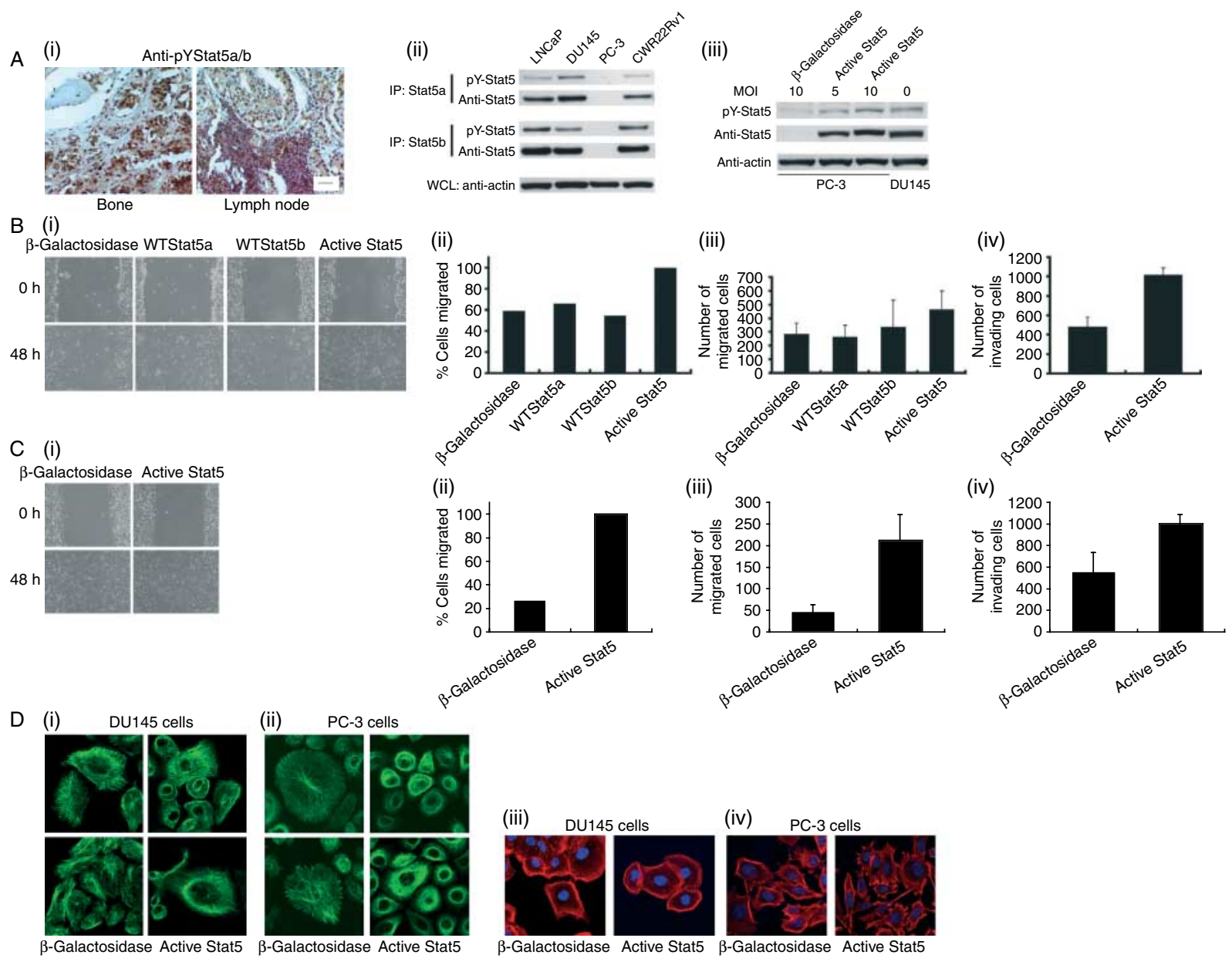

Figure 1 Stat5 is active in distant human prostate cancer metastases, and Stat5 induces migration and microtubule formation of human prostate cancer cells. (A) (i) Activation of Stat5 in prostate cancer metastases to bone (left panel) and lymph nodes (right panel) was analyzed by immunohistochemistry. Bar, $50 \mu \mathrm{m}$. (ii) Stat5a or Stat5b was immunoprecipitated (IP) from LNCaP, DU145, PC-3, and CWR22Rv1 cells, and was blotted with anti-phospho-Stat5a/b. Filters re-blotted with anti-Stat5ab mAb and whole cell lysates (WCLs) were immunoblotted with anti-actin pAb. (iii) PC-3 cells were infected with Ad-activeStat5 (Stat5aS710F) at different MOls as indicated. Whole cell lysates of PC-3 cells or DU145 cells were immunoblotted with anti-phospho-Stat5ab mAb, antiStat5ab pAb, or anti-actin pAb. DU145 (B) and PC-3 cells (C) were infected with Ad-wild-type Stat5a (AdWTStat5a), AdWTStat5b, Ad-activeStat5, or Ad- $\beta$-galactosidase (MOI 10). Identical scratches were made in parallel wells, and the cells were fixed and photographed, and wound sizes were measured at the indicated time points (i and ii). For Boyden chamber assays (iii and iv), DU145 (B) and PC-3 (C) cells were infected with AdWTStat5a, AdWTStat5b, Ad-activeStat5, or Ad- $\beta$-galactosidase (MOI 10). DU145

(A) and PC-3 (B) cell motility through uncoated filters (iii) or Matrigel-coated filters (iv) in three individual experiments (mean \pm s.D). (D) Stat5 induces rearrangement of the microtubule network of human prostate cancer cells while not affecting the organization of the actin cytoskeleton. DU145 and PC-3 cells infected with Ad-activeStat 5 or Ad- $\beta$-galactosidase were immunostained with $\alpha$-tubulin $\mathrm{mAb}$ ( $\mathrm{i}$ and ii) or rhodamine-conjugated phalloidin (iii and iv) using FITC-conjugated secondary antibodies.

$P=0.93)$, which led us to focus our subsequent assays on evaluating the effects of active Stat5 on metastatic behavior of prostate cancer cells. Inhibition of Stat5 by a dominant-negative (DN) mutant of Stat5 was not included in these experiments since inhibition of Stat5 causes massive apoptotic death of prostate cancer cells (Ahonen et al. 2003, Dagvadorj et al. 2008a), which would naturally inhibit cell migration. In addition to DU145 cells, Ad-activeStat5 increased migration of PC-3 cells (Fig. 1C, i and ii) and LNCaP cells
(Supplementary Figure 2, see section on supplementary data given at the end of this article) by approximately threefold versus $\beta$-galactosidase-expressing cells in wound-filling assays. In Boyden chamber assays, Ad-activeStat 5 stimulated the migration of PC-3 cells by fourfold $(P=0.01)$ and invasion by twofold $(P=0.057)$ compared with AdLacZ (Fig. 1C, iii and iv). In summary, these data demonstrate that active Stat5 increases motility of human prostate cancer cells in vitro. 
Table 1 Stat 5 activation in prostate cancer metastasis

\begin{tabular}{lcc}
\hline & $\begin{array}{c}\text { Number of } \\
\text { patients }\end{array}$ & Percentage \\
\hline $\begin{array}{l}\text { Prostate cancer metastases (lymph node metastases and } \\
\text { metastases to other organs) }\end{array}$ & 128 & 100 \\
Stat5a/b activation status & 50 & 39 \\
Negative & 78 & 61 \\
Positive & & \\
Prostate cancer metastases to regional lymph nodes & \\
Stat5a/b activation status & 59 & 100 \\
Negative & 11 & 19 \\
Positive & 48 & 81 \\
Prostate cancer metastases to bone & & \\
Stat5a/b activation status & 15 & 100 \\
Negative & 10 & 67 \\
Positive & 5 & 33 \\
Prostate cancer metastases to other organs & \\
Stat5a/b activation status & 54 & 100 \\
Negative & 29 & 54 \\
Positive & 25 & 46 \\
\hline
\end{tabular}

\section{Stat5 induces rearrangement of the microtubule network of prostate cancer cells}

We next examined whether organization of the microtubule network and actin polymerization would be affected by Stat5 in prostate cancer cells. In both DU145 and PC-3 cells, Ad-activeStat5 induced extensive polymerization of the microtubules with a formation of a dense meshwork around the nuclei compared with AdLacZ (Fig. 1D, i and ii) as has been described in other cell types (Goh et al. 1998, $\mathrm{Ng}$ et al. 2006, Hung et al. 2008). Organization of the actin cytoskeleton was not altered by active Stat5 (Fig. 1D, iii and iv). These data suggest that active Stat5 expression results in increased polymerization of the microtubule network in human prostate cancer cells.

\section{Active Stat5 suppresses cell surface E-cadherin expression in prostate cancer cells, while it increases heterotypic adhesion of prostate cancer cells to endothelial cells}

The levels of cell surface E-cadherin expression were highest in LNCaP cells, while the E-cadherin levels in DU145 and PC-3 cells were very low compared with LNCaP cells with the methodology used (Fig. 2A, i). Thus, we were able to study the effects of Stat5 on prostate cancer cell surface E-cadherin only in $\mathrm{LNCaP}$ cells. Active Stat5 induced a significant decrease in cell surface E-cadherin in LNCaP cells in $48 \mathrm{~h}$ (Fig. 2A, ii). To verify the immunohistochemistry data, cell surface proteins were first biotinylated, and E-cadherin was subsequently immunoprecipitated from LNCaP cell lysates using anti-E-cadherin antibody, followed by detection with streptavidin-conjugated HRP (Fig. 2A, iii). Ad-activeStat5 in $\mathrm{LNCaP}$ cells led to marked decrease in E-cadherin on the cell surface within $48 \mathrm{~h}$ (Fig. 2A, ii) in western blotting. This was related to active Stat5-induced migration of LNCaP cells (Supplementary Figure 1). The overall levels of E-cadherin did not change markedly in response to elevated active Stat5 expression in LNCaP cells (Fig. 2A, iv). In summary, these results indicate that active Stat5 expression in prostate cancer cells is associated with a decrease in the cell surface E-cadherin expression.

The initial arrest and attachment of cancer cells to vascular endothelium precede their extravasation from the blood stream, and are crucial steps in the tumor metastatic cascade. First, we determined the effect of active Stat5 on the adhesion of DU145, PC-3, and LNCaP prostate cancer cells to vascular endothelial cells. Expression of active Stat5 led to a fourfold increase in the binding of DU145 cells (Fig. 2B, i; $P=0.0022)$ to HUVECs, and to a ninefold or twofold increase in the binding of PC-3 and LNCaP cells respectively (Fig. 2B, ii and iii; $P<0.001$ ) compared with the controls. Secondly (Fig. 2B, iv-vi), we tested the effect of active Stat5 on the binding of human prostate cancer cells to HBMECs (Lehr \& Pienta 1998, Bocchinfuso et al. 2000). Active Stat5 increased the binding of DU145 cells to HBMECs by $70 \%$ $(P=0.0022)$, PC-3 cells by $40 \%(P=0.10)$, and LNCaP cells by $60 \%$ (Fig. 2B). These results suggest that Stat5 activation is associated with decreased levels of prostate cancer cell surface E-cadherin, while heterotypic adhesion of DU145 and PC-3 cells to human endothelial cells is increased by active Stat5.

\section{Stat5 induces experimental metastases of DU145 cells in nude mice}

To determine the effects of active Stat5 on metastatic dissemination of human prostate cancer cells in an in vivo experimental metastases assay, Ad-activeStat5 or AdLacZ-infected DU145 cells (MOI 10) were injected into athymic nude mice through the tail veins $24 \mathrm{~h}$ after the adenoviral gene delivery. The lungs were harvested after 8 weeks, and were scored for surface lung metastases. The number of lung metastases in mice that were injected with DU145 cells expressing active Stat5 increased by $\sim 11$-fold $($ mean $=105$, S.E.M. $=29.8)$ versus mice injected with $\beta$-galactosidase-expressing DU145 cells (mean $=9$, S.E.M. $=4.9, P=0.016$; Fig. 2 C). This is the first demonstration that Stat5 increases the intrinsic ability 

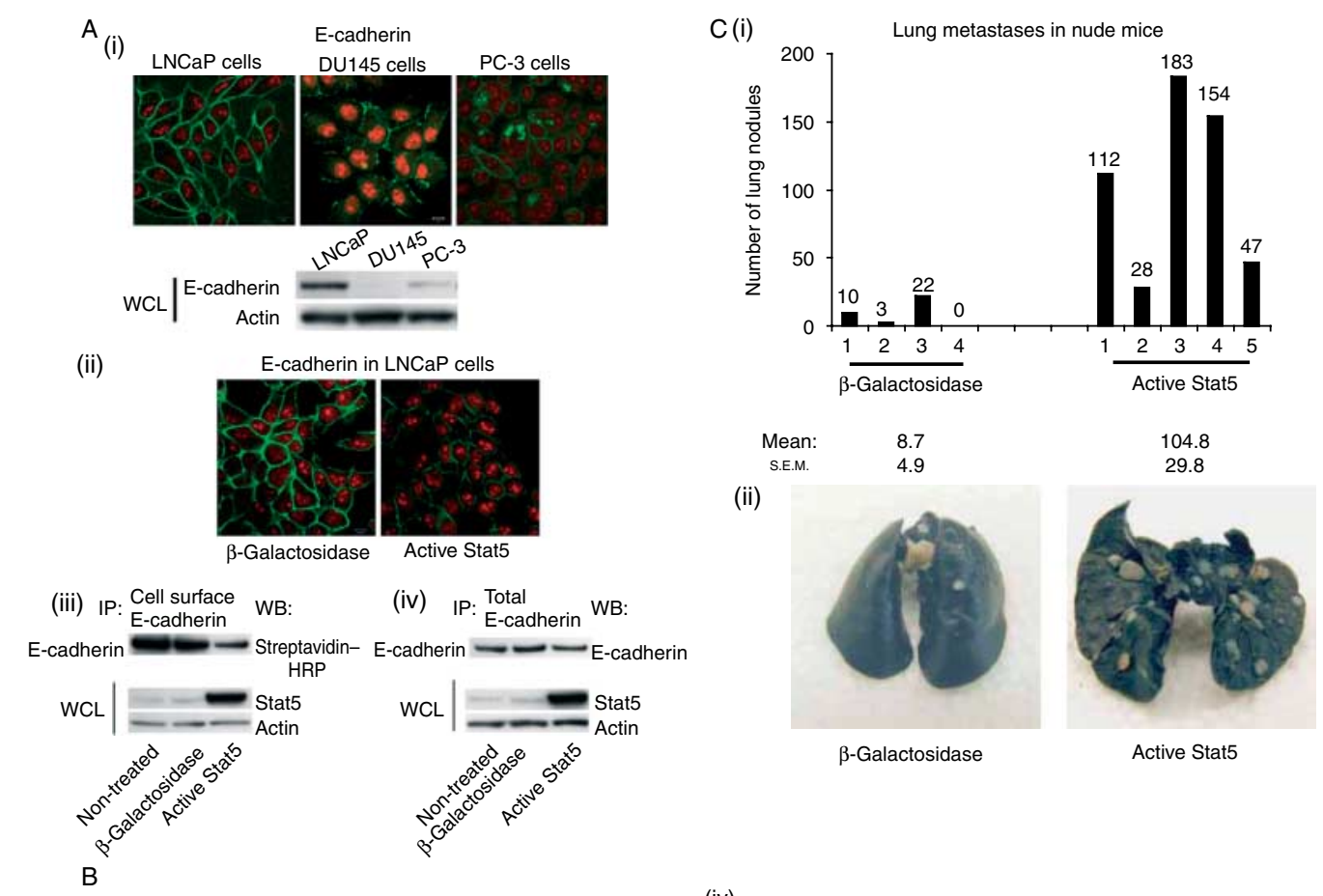

(iv) IP: Total E-cadherin WB:
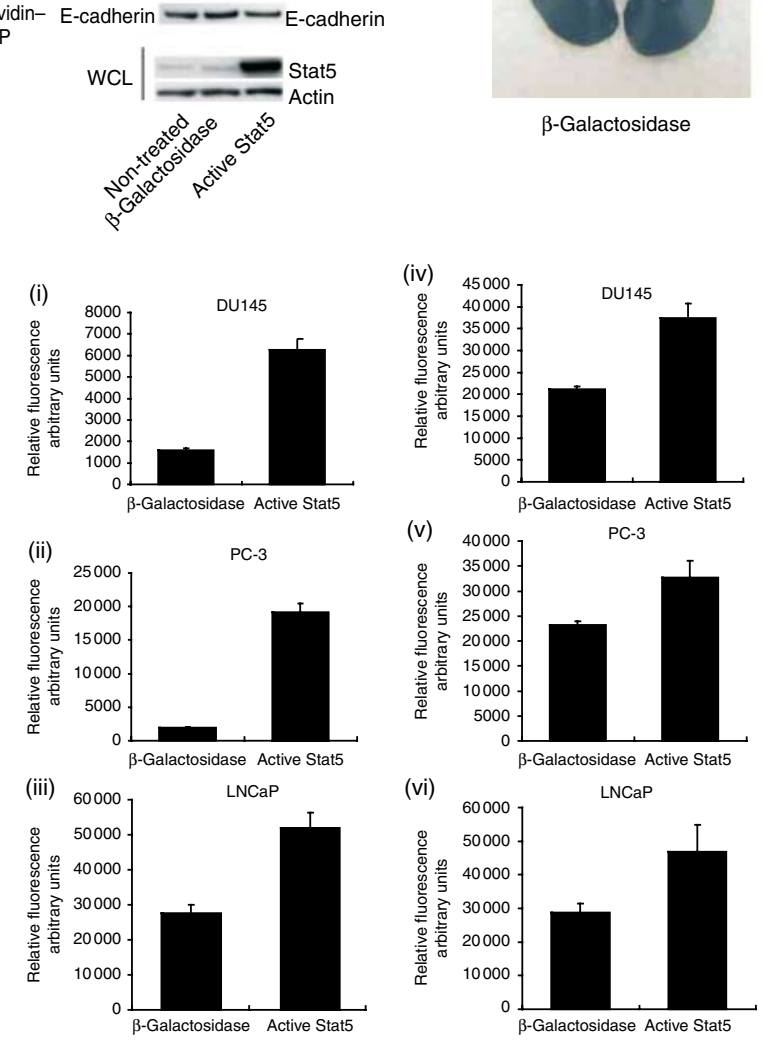

Figure 2 Active Stat5 regulates cell surface E-cadherin expression and heterotypic adhesion of prostate cancer cells, and Stat5 induces experimental prostate cancer metastases to the lungs of nude mice. (A) (i) LNCaP, DU145, and PC-3 cells were immunostained for E-cadherin using FITC-conjugated secondary antibodies (green). DNA was stained with propidium iodide (red). Total E-cadherin levels in LNCaP, DU145, and PC-3 cells were determined by immunoblotting WCLs with anti-E-cadherin mAb (lower panel). (ii) LNCaP cells were infected with Ad-activeStat5 or Ad- $\beta$-galactosidase (MOI 10), and after $48 \mathrm{~h}$, the cells were immunostained for E-cadherin as described above. (iii) LNCaP cells were biotinylated, and E-cadherin was immunoprecipitated (IP) and detected by western blotting (WB) as a $120 \mathrm{kDa}$ protein using streptavidin-HRP (upper panel). WCLs of the same samples were immunoblotted with anti-Stat $5 \mathrm{a} / \mathrm{b}$ and anti-actin antibodies. (iv) Total cellular levels of $\mathrm{E}$-cadherin were determined by

immunoprecipitation of parallel samples with E-cadherin mAb and immunoblotting with anti-E-cadherin mAb. (B) DU145, PC-3, and LNCaP cells were infected with Ad-activeStat5 or Ad- $\beta$-galactosidase (MOI 10). After $72 \mathrm{~h}$, the cells were stained with a fluorescent dye and allowed to adhere to HUVECs (i-iii) or HBMECs (iv-vi), and the adhered cells were quantitated. (C) Active Stat5 increases metastases formation of DU145 human prostate cancer cells in the lungs of athymic nude mice. Mice were injected with DU145 cells infected with Ad-activeStat5 or Ad- $\beta$-galactosidase (MOI 10) through the tail vein. The lungs were scored for surface metastases (i). Representative photographs of India Ink-stained lungs (ii). 
of prostate cancer cells to metastasize in vivo. The fact that the metastatic potential of DU145 cells in animal models has been shown to be overall somewhat weak (Nemeth et al. 1999, Timar et al. 2000, Singh et al. 2006) underscores the importance of Stat5 induction of DU145 cell metastases formation in nude mice.

\section{Stat5-regulated genes in human prostate cancer cells}

We inhibited Stat5 expression $48 \mathrm{~h}$ prior to the gene expression analysis by Stat $5 \mathrm{a} / \mathrm{b}$ siRNA using scrambled siRNA as the control (Fig. 3A) in DU145 cells. Overall, 777 genes were differentially expressed between control siRNA and Stat5 siRNA groups in DU145 cells using FDR $<0.01$ on the full dataset of 15992 genes (Supplementary Table 1). To define functional groups within the Stat5-regulated genes, we used the descriptions from the GO annotations as our tool. The GO is constructed in a hierarchical manner with categories corresponding to each GO identifier (GO ID) being potentially subdivided into more precise subcategories, each with its own GO ID. 'The metastasis group' of genes was defined as a set of 88 GO IDs and their subcategories (a total of 1975 GO IDs) corresponding to genes encoding proteins related to metastases processes. This resulted in 3234 unique metastases-related genes out of 15992 unique annotated genes on the chip (Supplementary Table 2, see section on supplementary data given at the end of this article). The apoptosis-related genes as well as the proliferation-related genes were defined similarly. Out of the 777 genes that were differentially expressed between control siRNA and Stat5 siRNA groups in DU145 prostate cancer cells, $163(21 \%)$ were from the 'metastasis group' of genes, 30 (3.9\%) were from the 'apoptosis group' of genes, and 61 (7.9\%) were from 'the proliferation group' of genes (Fig. 3B).

A heatmap based on a hierarchical clustering of the expression values of 50 most differentially expressed genes (as determined by the smallest $P$ values) related to metastases processes (Fig. 3C). In the heatmap, red represents higher expression, and green represents lower expression, and the genes (rows) are re-ordered based on a hierarchical clustering using the correlation metric. Cytokines and cytokine receptors (interferon- $\alpha,-\beta$, and $-\omega$ receptor 1 ; interleukin 31 receptor $A$; and erythropoietin), vascular endothelial growth factor $\mathrm{C}$ (VEGF-C), and collagen types VI and XII were identified among the metastases-related genes regulated by Stat5 in prostate cancer cells (Supplementary Table 2). In summary, the majority of Stat5-regulated genes in DU145 prostate cancer cells were metastases related.

\section{Stat5 activation and heterotypic adhesion of prostate cancer cells are rather related to Src activation than to Jak2 activation}

To identify the kinases involved in Stat5a/b activation and heterotypic adhesion of prostate cancer cells to endothelial cells, we focused first on determining the significance of Jak2 as the activator of Stat5a/b in prostate cancer cells. CWR22Rv (i), DU145 (ii), and LNCaP (iii) cells (Fig. 4A) were infected with AdDNJak2 or AdWTJak2 (MOI 10). In CWR22Rv cells, tyrosine phosphorylation of Stat $5 \mathrm{a} / \mathrm{b}$ was almost completely inhibited in cells expressing DNJak2, as we have shown previously (Li et al. 2004) demonstrating that our AdDNJak2 was functional (positive control). While WTJak2 increased Stat5a/b activation in all the three cell lines, DNJak2 was unable to block Stat5a/b activation in DU145 and LNCaP cells, suggesting that also kinases other than Jak2 are involved in the regulation of $S t a t 5 \mathrm{a} / \mathrm{b}$ activity in various human prostate cancer cells.

In several different cell types, Rho GTPases mediate serine and tyrosine phosphorylation and nuclear translocation of Stats (Simon et al. 2000, Aznar et al. 2001), and Rho family GTPases regulate the organization of microtubules in migratory cells (Yamazaki et al. 2005). When DU145 cells were treated with increasing concentrations of pharmacological RhoA inhibitor (Y27632) and a Rac1 inhibitor (553502) for $72 \mathrm{~h}$ (Fig. 4B), there were no differences in Stat5a/b activation levels associated with the inhibition of RhoA or Rac1 in DU145 or LNCaP cells (Fig. 4B).

To examine the involvement of Src activity (Ozawa et al. 2008, Yang et al. 2009) on Stat5a/b activation in prostate cancer cells, DU145 and LNCaP cells (Fig. 4C) were treated for 72 or $48 \mathrm{~h}$ with a specific Src inhibitor PP2 using PP3 as the control. Inhibition of Src kinases led to a dose-dependent inhibition of both Stat5a and Stat5b tyrosine phosphorylation in both DU145 and LNCaP cells. Inhibition of Src kinases by the Src inhibitor PP2 resulted in decreased binding of DU145 cells to HUVECs $(P=0.0022)$ and HBMECs $(P=0.01$; Fig. 4D). These results conclude that $S t a t 5 a / b$ activity related to metastatic behavior of human prostate cancer cells is mediated by Src kinases rather than by Jak 2 .

\section{Discussion}

Understanding the cellular and molecular basis of the metastases process of prostate cancer is fundamental for the identification of new therapeutic target proteins for disseminated prostate cancer. We have shown that 
A

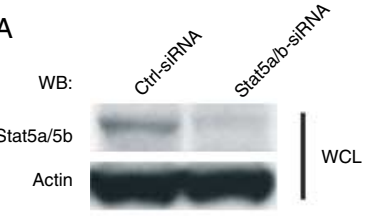

C

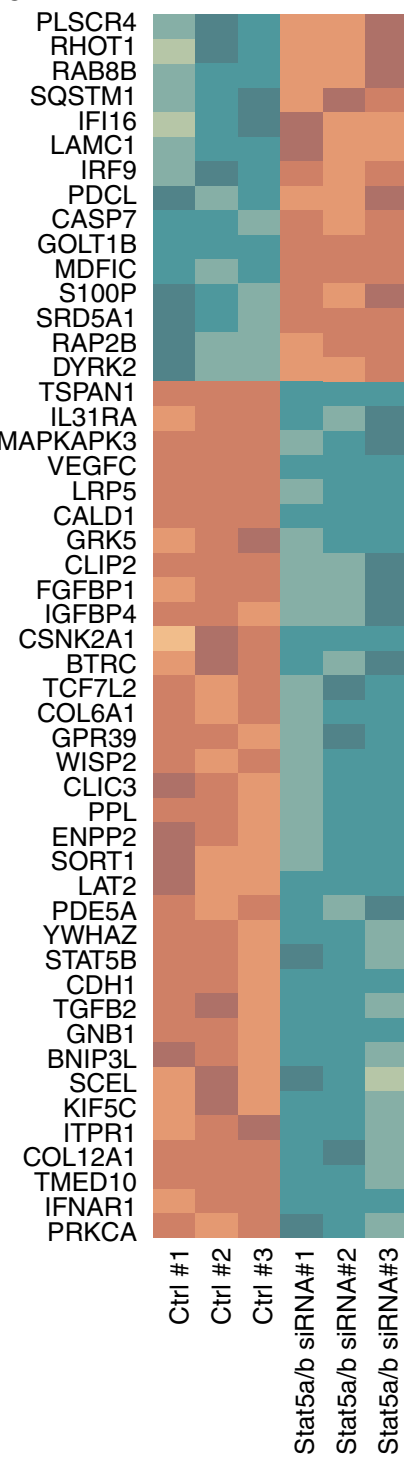

B

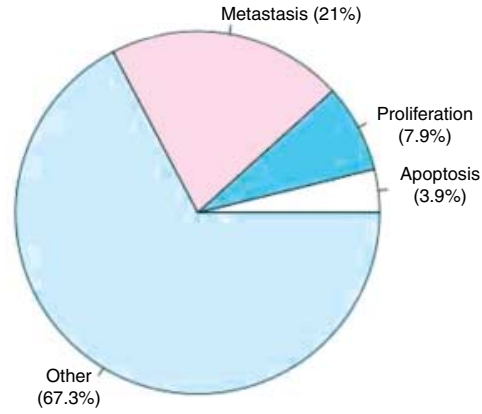

Phospholipid scramblase 4

Ras homolog gene family, member T1

RAB8B, member RAs oncogene family

Sequestosome 1

Interferon, $\gamma$-inducible protein 16

Laminin, $\gamma 1$ (formerly LAMB2)

Interferon regulatory factor 9

Phosducin-like

Caspase 7, apoptosis-related cysteine peptidase

Golgi transport 1 homolog B (S. cerevisiae)

MyoD family inhibitor domain containing

S100 calcium binding protein $P$

Steroid-5- $\alpha$-reductase, $\alpha$ polypeptide 1

RAP2B, member of RAS oncogene family

Dual-specificity throsine-( $Y$ )-phosphorylation regulated kinase 2

Tetraspanin 1

Interleukin 31 receptor $A$

Mitogen-activated protein kinase-activated protein kinase 3

Vascular endothelial growth factor $\mathrm{C}$

Low density lipoprotein receptor-related protein 5

Caldesmon 1

G protein-coupled receptor kinase 5

CAP-GLY domain containing linker protein 2

Fibroblast growth factor binding protein 1

Insulin-like growth factor binding protein 4

Casein kinase 2, $\alpha 1$ polypetide

$\beta$-Transducin repeat containing

Transcription factor 7-like 2 (T-cell specific, HMG-box)

Collagen, type VI, $\alpha 1$

G protein-coupled receptor 39

WNT1 inducible signaling pathway protein 2

Chloride intracellular channel 3

Periplakin

Ectonucleotide pyrophosphatase/phosphodiesterase 2 (autotaxin)

Sortilin 1

Linker for activation of $T$ cells family, member 2

Phosphodiesterase 5A, cGMP-specific

Throsine 3-monooxygenase/tryptophan 5-monooxygenase activation protein, zeta polypeptide

Signal transducer and activator of transcription $5 \mathrm{~B}$

Cadherin 1, type 1, E-cadherin (epithelial)

Transforming growth factor, $\beta 2$

Guanine nucleotide binding protein ( $G$ protein), $\beta$ polypeptide 1

BCL2/adenovirus E1B $19 \mathrm{kDa}$ interacting protein 3-like

Sciellin

Kinesin family member $5 \mathrm{C}$

Inositol 1,4,5-triphosphate receptor, type 1

Collagen, type XII, $\alpha 1$

Transmembrane emp24-like trafficking protein 10 (yeast)

Interferon $(\alpha, \beta$ and $\gamma)$ receptor 1

Protein kinase C, $\alpha$

Figure 3 Identification of Stat5-regulated genes in human prostate cancer cells. (A) DU145 cells were transfected with Stat5a/b siRNA or scrambled siRNA as control. After $48 \mathrm{~h}$, cell lysates were immunoblotted with anti-Stat5a/b mAb and re-blotted with anti-actin antibody. (B) Transcriptional profiles of Stat5-regulated genes in DU145 prostate cancer cells related to metastases, proliferation, or apoptosis out of a total of 777 genes that were differentially expressed between control siRNA and Stat5 siRNA using false discovery rate $<0.01$ on the full dataset of 15992 genes. (C) A heatmap showing the top 50 differentially expressed genes by Stat5 (determined by the smallest $P$ values) of the metastases-related group of genes. In the heatmap, red represents higher expression, whereas green represents lower expression. 

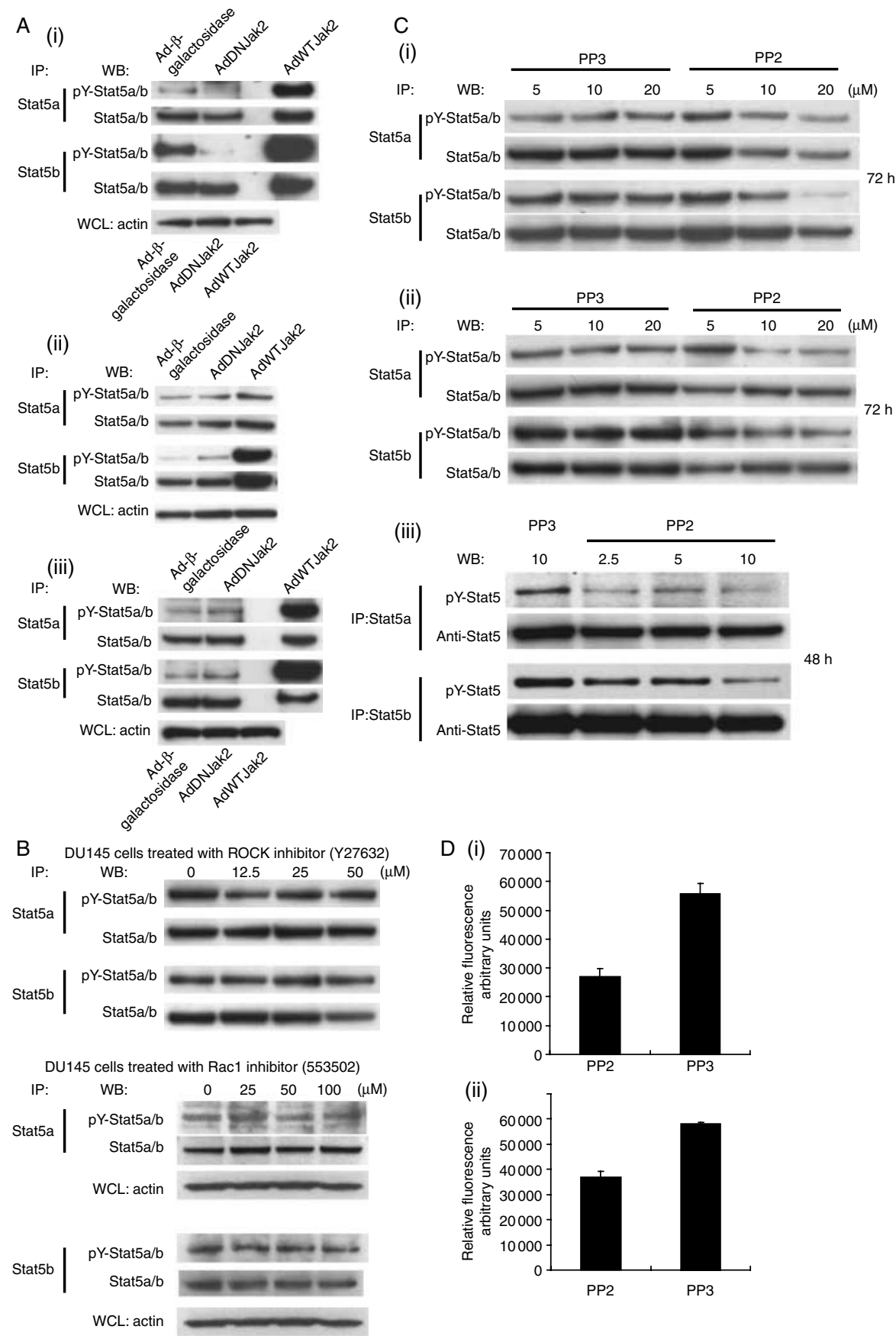

Figure 4 Stat5 activation and heterotypic adhesion of prostate cancer cells were related to Src kinase activation rather than to Jak2 activation. (A) CWR22Rv1 (i), DU145 (ii), and LNCaP (MOI 10) (iii) cells were infected with AdDNJak2, AdWTJak2, or AdLacZ for $48 \mathrm{~h}$, after which, immunoprecipitated Stat5a and Stat5b were immunoblotted with anti-phospho-Stat5a/b mAb, and the filters were re-blotted with anti-Stat5a/b mAb. WCLs of the same samples were blotted for actin. (B) DU145 cells were treated with pharmacological inhibitors of RhoA (Y27632) and Rac1 (553502) for $48 \mathrm{~h}$. Stat5a and Stat5b were immunoprecipitated and immunoblotted with anti-phospho-Stat5a/b mAb and anti-Stat5a/b mAb. (C) DU145 (i) and LNCaP (ii and iii) cells were treated with pharmacological Src inhibitor PP2 for 72 or $48 \mathrm{~h}$ (iii) using PP3 as a control compound. Levels of active Stat5a and Stat5b were analyzed by immunoprecipitation and western blotting as described above. (D) Pharmacological inhibition of Src kinases attenuates heterotypic adhesion of DU145 cells to endothelial cells. DU145 cells were treated with PP2 (20 $\mu$ M) using PP3 as the control for $72 \mathrm{~h}$, after which, the cells were stained with a fluorescent dye and allowed to bind to HUVECs (i) or HBMECs (ii) for 60 min before quantitation of the adhered cells. 
Stat5 is activated in the majority of distant metastases of clinical human prostate cancers. Active Stat5 increased metastases formation by 11-fold in an in vivo prostate cancer experimental metastases assay. The molecular mechanisms underlying Stat5induced experimental metastases formation likely include Stat5-stimulated migration and heterotypic adhesion of human prostate cancer cells, while active Stat5 suppressed cell surface E-cadherin expression in prostate cancer cells. Gene expression profiling indicated that $21 \%$ of Stat5-regulated genes in prostate cancer cells were related to metastases, while $7.9 \%$ were related to proliferation and 3.9\% to apoptosis.

This is the first study to show Stat5 induction of prostate cancer metastases formation in nude mice in an experimental metastases model, which is the key finding of the work presented here. This result is important because in vitro observations on invasiveness or migration of cells in culture do not necessarily translate into cells having the ability to metastasize in vivo. Inoculation of cancer cells into tail veins is known to result in the development of metastases in the lungs of mice, which contain the first capillary bed the cancer cells encounter. Stat5-induced metastases formation in the lungs of athymic nude mice may result from increased prostate cancer cell survival and avoidance of anoikis both in circulation and in the distant sites of metastases. This concept is supported by our findings showing that Stat5 is critical for the survival of human prostate cancer cell lines in vitro and for xenograft prostate cancer growth in vivo (Ahonen et al. 2003, Dagvadorj et al. 2008a).

An alternative explanation for increased lung metastases formation after tail vein injections is that Stat5 induces extravasation of prostate cancer cells from the capillaries to the lung tissue. This concept is supported by our finding of active Stat5-induced migration of DU145 and PC-3 human prostate cancer cells in wound-filling and Boyden chamber assays. Moreover, active Stat 5 induced heterotypic adhesion of human prostate cancer cells to endothelial cells. We have demonstrated that active Stat5 increased adhesion of human prostate cancer cells not only to HUVECs but also to HBMECs (Lehr \& Pienta 1998, Bocchinfuso et al. 2000), which represent a more relevant model system for studies on the regulation of heterotypic adhesion of prostate cancer cells. Initial docking of cancer cells to the endothelium is mediated by carbohydrate recognition. The cytokines expressed by cancer cells induce the expression of adhesion molecules in both endothelium and cancer cells which reinforce the initial adhesive bonds (Orr et al. 2000, Miles et al. 2008). Gene expression profiling of Stat5-regulated genes in DU145 cells included a number of cytokines and cytokine receptors, VEGF-C, and integrin ligands. Future studies should focus on Stat5 regulation of proteins mediating prostate cancer cell interactions with endothelial cells such as integrins and their ligands and selectin receptors CD44 and CD44v4 (Orr et al. 2000, Miles et al. 2008). Stat5 phosphorylation in DU145 cells and heterotypic adhesion of prostate cancer cells to endothelial cells were inhibited by a Src inhibitor, suggesting Src kinases as another class of Stat5-activating kinases in human prostate cancer cells in addition to Jak2, which we have described in CWR22Rv1 cells (Li et al. 2004). Intracardiac inoculation of human prostate cancer cells into nude mice will establish physiologically more relevant patterns of prostate cancer cell colonization regulated by Stat5. Orthotopic prostate cancer metastases studies would determine whether Stat5 will influence the metastases process initiating from prostate and including not only the local invasion but also the steps required for the formation of distant metastases.

Active Stat5 decreased cell surface E-cadherin expression in human prostate cancer cells. This is in agreement with our previous findings associating active Stat5 with loss of cell surface E-cadherin in 356 clinical prostate cancers (Li et al. 2005). E-cadherin, a transmembrane glycoprotein, is linked to the actin cytoskeleton via catenins to regulate tissue architecture and cell-cell adhesion structures (Nagafuchi et al. 1987). The inhibitory effect of Stat5 on the cell surface E-cadherin levels is likely due to decreased stabilization of E-cadherin on the cell surface of prostate cancer cells (Jeanes et al. 2008). This is supported by the western blotting data, which indicated no change in the total levels of E-cadherin protein, while the cell surface E-cadherin levels showed a robust decrease associated with active Stat5 expression. Future studies should include the analysis of Stat5 effects on other markers of epithelial-mesenchymal transition in prostate cancer cells such as proteins of the Twist family (Ansieau et al. 2008). In addition, future work should include studies testing whether Stat5 regulates cell surface E-cadherin levels in clinical prostate cancers obtained from patients. This could be achieved by, for example, using ex vivo 3D organ cultures as the experimental model system (Nevalainen et al. 1991, 1993, 1996, 1997a,b, Ahonen et al. 1999, 2002, Li et al. 2004).

In conclusion, this work provides the first evidence of the involvement of Stat 5 in metastatic progression of human prostate cancer cells in vivo. Stat5a/b may provide a therapeutic target protein specifically for advanced disseminated prostate cancer. 


\section{Supplementary data}

This is linked to the online version of the paper at http://dx. doi.org/10.1677/ERC-09-0328.

\section{Declaration of interest}

The authors declare that there is no conflict of interest that could be perceived as prejudicing the impartiality of the research reported.

\section{Funding}

Shared Resources of KCC are partially supported by NIH grant CA56036-08 (Cancer Center Support Grant, to KCC). This work was supported by ACS (RSG-04-196-01-MGO), DOD (W81XWH-05-01-0062 and W81XWH-07-1-0411), and NIH NCI (1RO1CA113580-01A1) grants.

\section{Acknowledgements}

We thank Dr Rui for providing AdDNJak2 and AdWTJak2, and Dr Almeida-Porada for providing the HBMECs.

\section{References}

Ahonen TJ, Harkonen PL, Laine J, Rui H, Martikainen PM \& Nevalainen MT 1999 Prolactin is a survival factor for androgen-deprived rat dorsal and lateral prostate epithelium in organ culture. Endocrinology 140 5412-5421.

Ahonen TJ, Harkonen PL, Rui H \& Nevalainen MT 2002 PRL signal transduction in the epithelial compartment of rat prostate maintained as long-term organ cultures in vitro. Endocrinology 143 228-238.

Ahonen TJ, Xie J, LeBaron MJ, Zhu J, Nurmi M, Alanen K, Rui H \& Nevalainen MT 2003 Inhibition of transcription factor Stat5 induces cell death of human prostate cancer cells. Journal of Biological Chemistry 278 27287-27292.

Ansieau S, Bastid J, Doreau A, Morel AP, Bouchet BP, Thomas C, Fauvet F, Puisieux I, Doglioni C, Piccinin S et al. 2008 Induction of EMT by twist proteins as a collateral effect of tumor-promoting inactivation of premature senescence. Cancer Cell 14 79-89.

Aznar S, Valeron PF, del Rincon SV, Perez LF, Perona R \& Lacal JC 2001 Simultaneous tyrosine and serine phosphorylation of STAT3 transcription factor is involved in Rho A GTPase oncogenic transformation. Molecular Biology of the Cell 12 3282-3294.

Benitah SA, Valeron PF, Rui H \& Lacal JC 2003 STAT5a activation mediates the epithelial to mesenchymal transition induced by oncogenic RhoA. Molecular Biology of the Cell 14 40-53.

Benjamini Y \& Hochberg Y 1995 Controlling the False Discovery Rate: A Practical and Powerful Approach to Multiple Testing. Journal of the Royal Statistical Society. Series B (Statistical Methodology) 57 289-300.
Bocchinfuso WP, Lindzey JK, Hewitt SC, Clark JA, Myers PH, Cooper R \& Korach KS 2000 Induction of mammary gland development in estrogen receptor-alpha knockout mice. Endocrinology 141 2982-2994.

Cheville JC, Tindall D, Boelter C, Jenkins R, Lohse CM, Pankratz VS, Sebo TJ, Davis B \& Blute ML 2002 Metastatic prostate carcinoma to bone: clinical and pathologic features associated with cancer-specific survival. Cancer 95 1028-1036.

Dagvadorj A, Collins S, Jomain JB, Abdulghani J, Karras J, Zellweger T, Li H, Nurmi M, Alanen K, Mirtti T et al. 2007 Autocrine prolactin promotes prostate cancer cell growth via Janus kinase-2-signal transducer and activator of transcription-5a/b signaling pathway. Endocrinology 148 3089-3101.

Dagvadorj A, Kirken RA, Leiby B, Karras J \& Nevalainen MT 2008a Transcription factor signal transducer and activator of transcription 5 promotes growth of human prostate cancer cells in vivo. Clinical Cancer Research 14 1317-1324.

Dagvadorj A, Tan SH, Liao Z, Cavalli LR, Haddad BR \& Nevalainen MT 2008b Androgen-regulated and highly tumorigenic human prostate cancer cell line established from a transplantable primary CWR22 tumor. Clinical Cancer Research 14 6062-6072.

Falcon S \& Gentleman R 2007 Using GOstats to test gene lists for GO term association. Bioinformatics 23 257-258.

Gentleman RC, Carey VJ, Bates DM, Bolstad B, Dettling M, Dudoit S, Ellis B, Gautier L, Ge Y, Gentry J et al. 2004 Bioconductor: open software development for computational biology and bioinformatics. Genome Biology 5 R80.

Goh EL, Pircher TJ \& Lobie PE 1998 Growth hormone promotion of tubulin polymerization stabilizes the microtubule network and protects against colchicineinduced apoptosis. Endocrinology 139 4364-4372.

Hung LY, Tseng JT, Lee YC, Xia W, Wang YN, Wu ML, Chuang YH, Lai CH \& Chang WC 2008 Nuclear epidermal growth factor receptor (EGFR) interacts with signal transducer and activator of transcription 5 (STAT5) in activating Aurora-A gene expression. Nucleic Acids Research 36 4337-4351.

Ihle JN 2001 The Stat family in cytokine signaling. Current Opinion in Cell Biology 13 211-217.

Irizarry RA, Hobbs B, Collin F, Beazer-Barclay YD, Antonellis KJ, Scherf U \& Speed TP 2003 Exploration, normalization, and summaries of high density oligonucleotide array probe level data. Biostatistics 4 249-264.

Jeanes A, Gottardi CJ \& Yap AS 2008 Cadherins and cancer: how does cadherin dysfunction promote tumor progression? Oncogene 27 6920-6929.

Lehr JE \& Pienta KJ 1998 Preferential adhesion of prostate cancer cells to a human bone marrow endothelial cell line. Journal of the National Cancer Institute 90 118-123. 
Li H, Ahonen TJ, Alanen K, Xie J, LeBaron MJ, Pretlow TG, Ealley EL, Zhang Y, Nurmi M, Singh B et al. 2004 Activation of signal transducer and activator of transcription 5 in human prostate cancer is associated with high histological grade. Cancer Research 64 4774-4782.

Li H, Zhang Y, Glass A, Zellweger T, Gehan E, Bubendorf L, Gelmann EP \& Nevalainen MT 2005 Activation of signal transducer and activator of transcription-5 in prostate cancer predicts early recurrence. Clinical Cancer Research 11 5863-5868.

Miles FL, Pruitt FL, van Golen KL \& Cooper CR 2008 Stepping out of the flow: capillary extravasation in cancer metastasis. Clinical \& Experimental Metastasis 25 305-324.

Moriggl R, Sexl V, Kenner L, Duntsch C, Stangl K, Gingras S, Hoffmeyer A, Bauer A, Piekorz R, Wang D et al. 2005 Stat5 tetramer formation is associated with leukemogenesis. Cancer Cell 7 87-99.

Nagafuchi A, Shirayoshi Y, Okazaki K, Yasuda K \& Takeichi M 1987 Transformation of cell adhesion properties by exogenously introduced E-cadherin cDNA. Nature 329 341-343.

Nemeth JA, Harb JF, Barroso U Jr, He Z, Grignon DJ \& Cher ML 1999 Severe combined immunodeficient-hu model of human prostate cancer metastasis to human bone. Cancer Research 59 1987-1993.

Nevalainen MT, Valve EM, Makela SI, Blauer M, Tuohimaa PJ \& Harkonen PL 1991 Estrogen and prolactin regulation of rat dorsal and lateral prostate in organ culture. Endocrinology 129 612-622.

Nevalainen MT, Härkönen PL, Valve EM, Ping W, Nurmi M \& Martikainen PM 1993 Hormone regulation of human prostate in organ culture. Cancer Research 53 5199-5207.

Nevalainen MT, Valve EM, Ingleton PM \& Harkonen PL 1996 Expression and hormone regulation of prolactin receptors in rat dorsal and lateral prostate. Endocrinology 137 3078-3088.

Nevalainen MT, Valve EM, Ahonen T, Yagi A, Paranko J \& Harkonen PL 1997a Androgen-dependent expression of prolactin in rat prostate epithelium in vivo and in organ culture. FASEB Journal 11 1297-1307.

Nevalainen MT, Valve EM, Ingleton PM, Nurmi M, Martikainen PM \& Harkonen PL $1997 b$ Prolactin and prolactin receptors are expressed and functioning in human prostate. Journal of Clinical Investigation 99 618-627.

Nevalainen MT, Xie J, Bubendorf L, Wagner KU \& Rui H 2002 Basal activation of transcription factor signal transducer and activator of transcription (Stat5) in nonpregnant mouse and human breast epithelium. Molecular Endocrinology 16 1108-1124.
Nevalainen MT, Xie J, Torhorst J, Bubendorf L, Haas P, Kononen J, Sauter G \& Rui H 2004 Signal transducer and activator of transcription-5 activation and breast cancer prognosis. Journal of Clinical Oncology 22 2053-2060.

$\mathrm{Ng}$ DC, Lin BH, Lim CP, Huang G, Zhang T, Poli V \& Cao X 2006 Stat 3 regulates microtubules by antagonizing the depolymerization activity of stathmin. Journal of Cell Biology 172 245-257.

Onishi M, Nosaka T, Misawa K, Mui AL, Gorman D, McMahon M, Miyajima A \& Kitamura T 1998 Identification and characterization of a constitutively active STAT5 mutant that promotes cell proliferation. Molecular and Cellular Biology 18 3871-3879.

Orr FW, Wang HH, Lafrenie RM, Scherbarth S \& Nance DM 2000 Interactions between cancer cells and the endothelium in metastasis. Journal of Pathology 190 310-329.

Ozawa Y, Williams AH, Estes ML, Matsushita N, Boschelli F, Jove R \& List AF 2008 Src family kinases promote AML cell survival through activation of signal transducers and activators of transcription (STAT). Leukemia Research 32 893-903.

Simon AR, Vikis HG, Stewart S, Fanburg BL, Cochran BH \& Guan KL 2000 Regulation of STAT3 by direct binding to the Rac1 GTPase. Science 290 144-147.

Singh AS, Macpherson GR, Price DK, Schimel D \& Figg WD 2006 Evaluation of human fetal bone implants in SCID mice as a model of prostate cancer bone metastasis. Oncology Reports 15 519-524.

Smyth GK 2004 Linear models and empirical bayes methods for assessing differential expression in microarray experiments. Statistical Applications in Genetics and Molecular Biology 3 Article 3.

Tan SH, Dagvadorj A, Shen F, Gu L, Liao Z, Abdulghani J, Zhang Y, Gelmann EP, Zellweger T, Culig Z et al. 2008 Transcription factor Stat5 synergizes with androgen receptor in prostate cancer cells. Cancer Research $\mathbf{6 8}$ 236-248.

Timar J, Raso E, Dome B, Li L, Grignon D, Nie D, Honn KV \& Hagmann W 2000 Expression, subcellular localization and putative function of platelet-type 12-lipoxygenase in human prostate cancer cell lines of different metastatic potential. International Journal of Cancer 87 37-43.

Yamazaki D, Kurisu S \& Takenawa T 2005 Regulation of cancer cell motility through actin reorganization. Cancer Science 96 379-386.

Yang X, Qiao D, Meyer K \& Friedl A 2009 Signal transducers and activators of transcription mediate fibroblast growth factor-induced vascular endothelial morphogenesis. Cancer Research 69 1668-1677. 